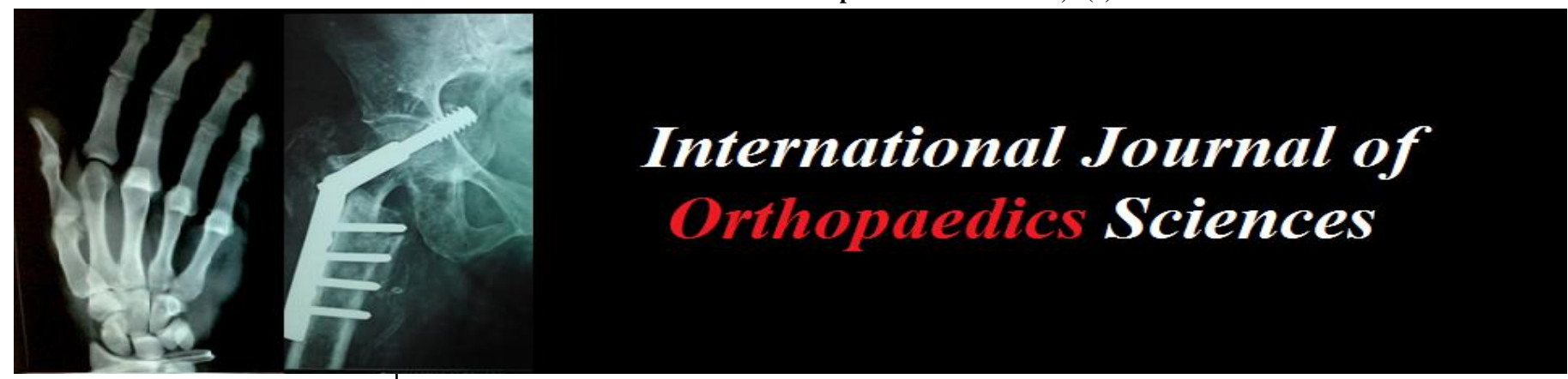

E-ISSN: 2395-1958

P-ISSN: 2706-6630

IJOS 2019; 5(4): 478-482

(C) 2019 IJOS

www.orthopaper.com

Received: 04-08-2019

Accepted: 06-09-2019

Dr. Johney Juneja

Fellow, Department of Joint

Replacement Orthopaedic

College Working/Studying

Palace, Lokmanya Hospital

Pune, Maharashtra, India

Dr. Narendra Vaidya

HOD, Joint Replacement and

Trauma Department, Lokmanya

Hospital Pune, Maharashtra,

India

Dr. Rakesh Patil

Consultant, Joint Replacement

and Trauma Department,

Lokmanya Hospital Pune,

Maharashtra, India
Corresponding Author: Dr. Johney Juneja

Fellow, Department of Joint Replacement Orthopaedic College Working/Studying Palace, Lokmanya Hospital Pune, Maharashtra, India

\section{To evaluate the strength of association between standard pre \& postoperative radiographic \& intraoperative navigation coronal alignment measurements}

\author{
Dr. Johney Juneja, Dr Narendra Vaidya and Dr. Rakesh Patil
}

DOI: $\underline{\text { https://doi.org/10.22271/ortho.2019.v5.i4i.1718 }}$

Abstract

Background: Precise pre- and postoperative measurements of limb alignment are necessary to plan and evaluate the success of TKA. There is a wealth of published literature attesting to the precision of intraoperative navigation systems over radiographic measurement as - gold standard for TKA alignment.

Aims and Objectives: (1) To evaluate the strength of the association between standard pre- and postoperative radiographic and intraoperative navigation alignment measurements; (2) To determine the potential sources of error inherent to the process of radiographic and navigated alignment assessment; and (3) To examine the subtle differences that exist between desired alignment (anteroposterior [AP] mechanical axis and clinically accepted alignment (final alignment after the bone cuts were made and implants placed) as assessed through an intraoperative navigation system.

Material and Methods: The study was conducted from April 2015 to May 2017. Data from a total of 60 consecutive total knee arthroplasties were included. Clinical measurement, radiographic measurement, computer navigation system measurement were carried out. BrainLab IV navigator was used in all cases. Results: Prior to surgery: The mean varus deformity as assessed on navigation was significantly lower in the navigation in comparison to the clinical assessment as well as by $\mathrm{x}$-ray $(p<0.05)$, while varus deformity assessment done clinically and by $\mathrm{x}$-ray was found to be comparable $(p>0.05)$.

After surgery: The mean deformity was significantly higher on clinical assessment and X-ray (valgus) in comparison to the navigation (varus) $(p<0.05)$. Mean valgus deformity clinically was more in comparison to the $\mathrm{X}$-ray $(p<0.05)$.

Conclusion: Advantages include improved restoration of joint line, reduction in peri-operative blood loss, better results with extra-articular deformities. Complications associated with navigation are rare. Better alignment improves functional outcome and long-term survival of prosthesis.

Keywords: Total knee arthroplasty, pre- and postoperative radiographic, intraoperative \& navigation

\section{Introduction}

Restoration of the mechanical alignment of the knee is one of the key criteria for a successful knee joint replacement. Alignment is almost exclusively measured from long-leg radiographs especially when cited to evaluate clinical and functional outcomes. However, other approaches to measuring alignment have been reported in the literature, including clinical examination, CT, MRI, short-leg radiographs, intraoperative supine fluoroscopy and intraoperative navigation. The accuracy of the above mentioned modalities for determining lower limb alignment have often been questioned, and currently no gold-standard exists. The long-leg radiograph (LLR) is traditionally measured on weight-bearing films including the hip, knee and ankle, with the patella facing forwards, and the X-ray beam parallel to the joint-line. The alignment is calculated as the difference between the femoral mechanical axis, defined by a line connecting the centre of the femoral head and the femoral intercondylar notch, and the tibial mechanical axis, being a line connecting the interspinous intercruciate midpoint of the tibial plateau and the midpoint of the tibial plafond.

There are three techniques to achieve accurate imaging modalities intraoperatively: (a) preoperative imaged technique - wherein information from CT/MRI of the knee and alignment about the anatomy of the knee is taken; (b) intraoperative imaging technique-wherein modified 
fluoroscopy is used for anatomical mapping of the knee during surgery, however, these are not commonly used in TKA and (c) Image-free technique - is the most recent technique used where an anatomical model that is fixed in the software is upgraded by the process of surface registration or bone morphing. However, the accuracy of this technique has not been reported yet. All these three techniques require the process of registration. Once registration is done, it is possible to proceed with operation.

The present study was carried out with an aim to compare the pre- and post-operative measurements of coronal alignment of the lower limb obtained from long-leg radiographs with the results obtained by computer navigation in patients undergoing primary TKR, with the following objectives: (a) to assess the accuracy of pre- and post-operative radiographs and intraoperative navigation alignment; (b) to determine the sources of error inherent in performing the radiographic and navigation alignment assessment; and (c) to examine the subtle differences that exist between desired alignment (anteroposterior [AP] mechanical axis and clinically accepted alignment (final alignment after the bone cuts were made and implants placed) as assessed through an intraoperative navigation system.

\section{Material and Methods}

The present prospective, observational study was conducted in Department of Orthopedics of Index Medical College, Hospital and Research Centre, Indore (M.P.) and Department of Joint Replacement, Lokmanya Hospital, Pune (M.S.) from April 2015 to November 2018. Forty knees undergoing total knee arthroplasties in the study were included.

All patients with age more than 45 years with primary osteoarthritis of the knee joint and rheumatoid arthritis of the knee, willing to provide their voluntary written informed consent to participate in the study were included, while patients with non-rheumatoid and/or non-osteoarthritis knee pathology, patients with complex primary total knee arthroplasty (range of motion $<500$, angular deformity $>100$, FFD $>30$, neurovascular problems), patients undergoing revision arthroplasty, patients with BMI $>40 \mathrm{~kg} / \mathrm{m} 2$ of ASA grade more than II, patients with Parkinsonism / latent or recent bone infection and those not giving consent for participation in the study were excluded.

A formal approval from the Institutional Ethics Committee was obtained prior to the conduct of the study and also before conducting any study related procedure, a voluntary written informed consent was obtained from the patient and/or his/her legally acceptable representative.

Enrolled patients underwent thorough history taking, clinical evaluation and available relevant radiological examination.

Detailed clinical examination included presence of tenderness, swelling or any abnormal mobility in the included knee. Active and passive range of motion, any flexion deformity or instability was noted. Knee circumference measured at mid-patellar level. Amount of varus / valgus deformity, flexion contracture and range of motion of the joints were noted along with any distal neurovascular deficit, if any. Relevant hematological investigations, preoperative anesthestic checkup and fitness as required from the physician and/or cardiologist was obtained prior to performing the surgery.

Computer Assisted Total Knee Arthroplasty (CATKA) was performed under spinal anesthesia with patient in supine position and under tourniquet control.

The measurements were performed by the senior surgeon or a trainee under his supervision. Demographic data and body mass indices were collected for all patients.

Patient was followed-up on Day 0 (operative day), Day 1-4, Day 5, Day 6-15, 3-6 weeks, 6 weeks-3 months and 3 months6 months, where patient was advised for postoperative rehabilitation and physiotherapy.

All the sutures were removed between Day $12-15^{\text {th }}$ from the surgery.

\section{Clinical measurements}

Clinical measurements were done on the day of admission with patient exposed from the waist down. Patient was then asked to assume usual stance to avoid excessive limb rotation. For coronal plane measurement, surface landmarks were used to identify the hip, knee and ankle. The midpoint between the anteriorsuperior iliac spine $\&$ the pubic tubercle was marked as the hip center. The center of knee as the point between the medial and lateral border of the knee. The point in the anterior ankle between the medial and lateral malleolus was marked as the center of the ankle. A long arm goniometer was used to measure the coronal lower limb alignment using these surface landmarks. For flexion deformity, the patient was viewed from the side and the long axis of the thigh and the leg were determined, and the angle between them measured with the goniometer.

\section{Radiographic measurements}

The pre-operative HKA radiographs, as stored in a Picture Archiving System (PACS, Kodak), were measured by two independent observers. The centre of the hip was identified using concentric circles (Fig. 1). ${ }^{[1]}$ The knee center was identified as the center of the line connecting the femoral trochlear midpoint and upper tibial midpoint. The ankle center was identified as the midpoint of the upper border of the talus [2]. The line connecting the three centers formed the coronal alignment of the knee (Fig. 2).

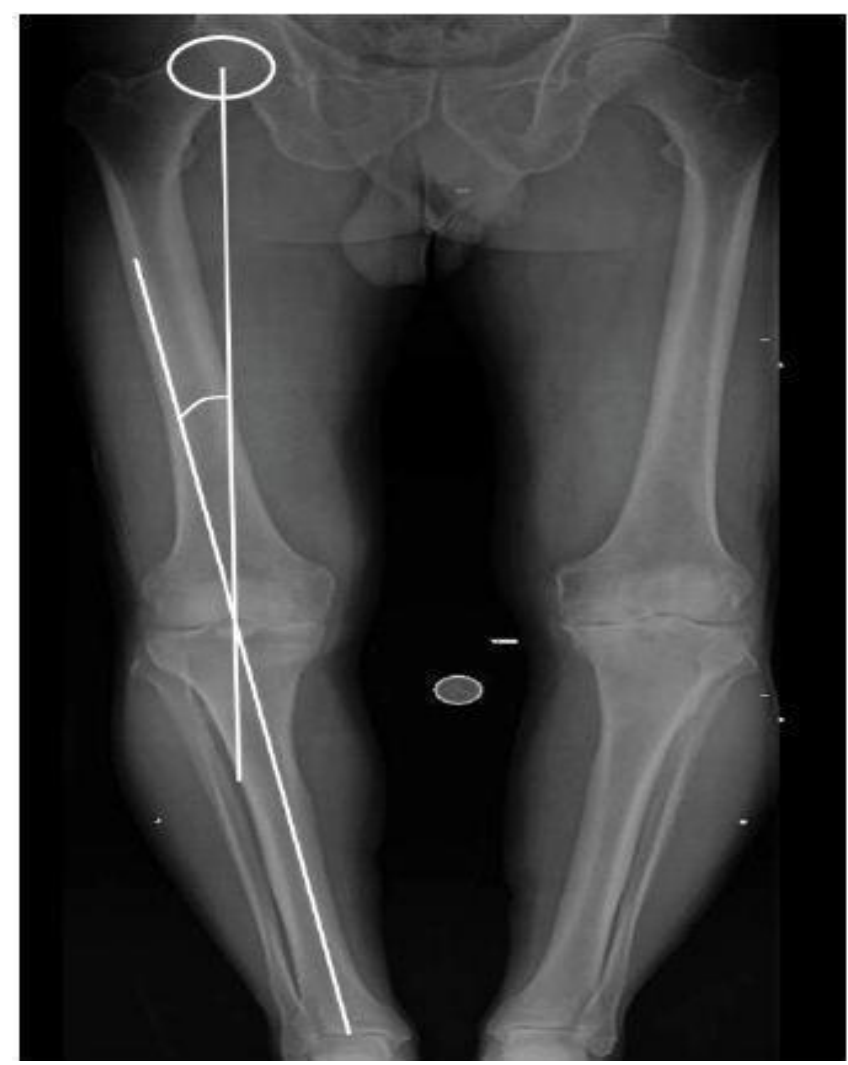

Fig 1: Measurement of mechanical axis with standing hip-kneeankle radiograph 


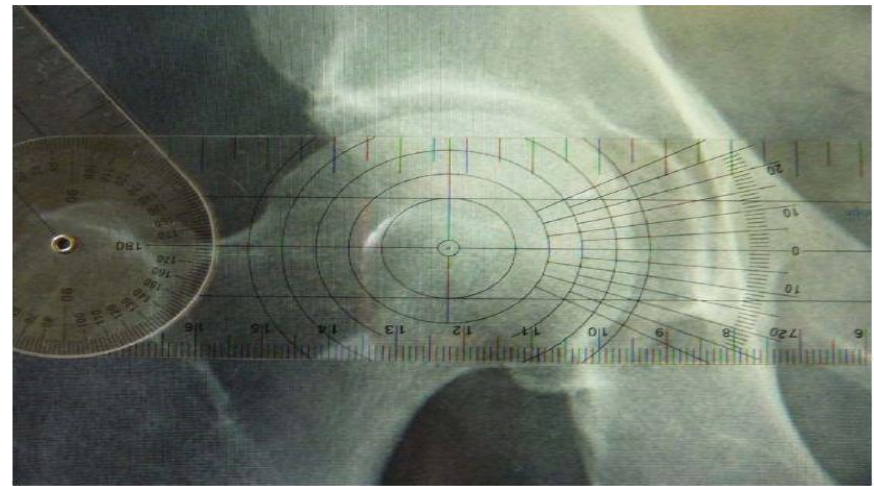

Fig 2: Concentric circles to locate hip centre

\section{Computer navigation system measurements}

Intra-operative (pre-implant) computer navigation measurements were performed using either the Stryker eNlite Navigation System, Stryker Navigation System II or Orthopilot (BBraun Aesculap, Tuttlingen, Germany) imagefree systems. The leg was registered as per system workflow after exposure to acquire the landmarks required by the software. The leg was then held in maximum extension and axial load was manually applied to the foot to simulate weight bearing. The coronal and sagittal alignments of the limb were recorded in the computer file (Fig. 2).

\section{Surgical Details}

After a median longitudinal skin incision had been made, medial parapatellar arthrotomy was performed and patellar resurfacing done. After the joint had been exposed, pins with passive reflective sensors (DRB) were implanted in the anteromedial region of the distal femur and proximal tibia, for the navigator to read. The reference points requested by the navigator were then gathered. The information relating to the patient's anatomy and joint ranges of motion were then input to the software. After data-gathering had been concluded, the patient's initial mechanical axis was informed by the system. Cuts were then made, firstly in the tibia, always orthogonal to its mechanical axis and without posterior inclination. Before femoral cuts were made, the ligaments were balanced in flexion and extension, by means of laminar tensioners under the control of the navigator on a millimeter scale. These data on the gap (space) in flexion and extension were filed by the system and registered as initial data. After this step, planning for the femoral cut was done. At this time, using the data on limb alignment, balancing of flexion and extension gaps, femoral rotation and femoral joint inclination, the size of the prosthesis and its best positioning could be defined, always in relation to the mechanical axis. After the femoral and tibial cuts had been made, the respective trial components were tested, and the quality of the limb alignment in relation to the mechanical axis in the coronal and sagittal planes and adequacy of the balancing for the planned implants were again investigated by means of the navigator. All these data were recorded and stored. All the components were then placed appropriately and cemented. The data were again gathered, confirmed and, at this time, recorded as final. This concluded the navigated stage of the procedure and the surgical site was then closed. The data were duly recorded and subsequently compared in order to produce this study. All the operations were performed by the same surgeon.

The implants used were the PFC SIGMA Depuy PS prosthesis and the navigator used was the BrainLab IV in all cases. No intra medullary guides were used. The bone cuts and all the other procedures were navigation assisted.
The implants used were the PFC SIGMA Depuy PS prosthesis and the navigator used was the BrainLab IV in all cases. No intra medullary guides were used. The bone cuts and all the other procedures were navigation assisted.

\section{Extraction method for data and variables}

To conduct the present study, the mechanical axis of the lower limb (initial and final) and the flexion and extension gaps (lateral and medial) were measured intra operatively by means of navigation, as described above. All the data were obtained preoperatively and postoperatively by clinically, radiographically and by navigation before and after performing the bone cuts and/or positioning the prosthesis, taking the first to be "initial" and the second, "final". The alignment and balancing obtained intraoperatively were verified based on the mechanical axis of the lower limbs and the final flexion and extension gaps (in millimeters), respectively. Knees were considered to be well aligned if, after arthroplasty, they presented not more than $3^{0}$ of deviation in the coronal plane.

\section{Financial input and funding}

As in the present study, the procedure, techniques and the drugs were used routinely; also, no additional tests/procedure was carried out on the patient for the specific requirement of the study. Hence, there was no burden either on the patient or on the institute. Also the present study is not being sponsored by any pharmaceutical company or any institution. All the study related expenses were borne by the investigator himself.

\section{Ethical consideration}

The subjects were explained about the study and about the procedure that will be adopted during the surgery. The drugs used in the study are already marketed drug and no new drug is being tested nor is this a clinical trial. The operations were conducted only after getting the voluntary high risk consent signed by the patient and/or his legally acceptable representative, which is the routine procedure followed by the hospital, for all the patients undergoing any major/minor surgical procedure. Apart from this consent, the subject also had to sign the informed consent form voluntarily by giving his/her consent for participation in this study. Prior to the initiation of the study in the institution, a proper approval from the ethical committee and the scientific committee was obtained.

\section{Statistics analysis}

The Bland Altman plot is drawn for by plotting the differences on the $\mathrm{Y}$-axis and averages on the $\mathrm{X}$-axis and the analysis based on this plot. Mean comparisons between the two groups was done using Unpaired ' $t$ ' test. A $p$ value of $<$ 0.05 was taken as statistically significant.

\section{Results}

Most of the time surgeons record the clinical deformity measurements in the patient notes. This forms a part of preoperative planning, and post-operatively it becomes an important reference point against which improvement in alignment is compared and is therefore vital in patient evaluation. It may also serve as a legal record in cases of litigation. In our study we found clinical measurement to be an unreliable method of deformity assessment.

Precise pre-and postoperative measurements of limb alignment are necessary to plan, perform, and evaluate the outcomes of TKA. Under ideal circumstances standard 
radiographs can in normal patients with minimal deformity be accurate ${ }^{[3,4]}$. However, these required conditions are difficult to achieve and are not applicable to patients with extremitydeforming osteoarthritis. Intraoperative navigation has an accuracy to within $1^{\circ},[5,6,7]$ and may allow objective assessment of the accuracy and reproducibility of radiographic measurement of TKA components and alignment. The purpose of our study, therefore, was to develop a more complete understanding of the relationship that exists between measurements performed using clinical measurement, standard radiographs and those obtained with an intraoperative navigation system. For analysis, Bland Altman Plot was used.

\section{Before the surgery}

The mean value obtained on the clinical assessment was -18.0 \pm 13.5 , while that obtained on the navigation was $-9.28 \pm$ 8.66. The difference was found to be statistically significant $(P<0.05)$ with a higher mean as measured clinically in comparison to the lower mean as seen on the navigation. The variance as seen on the navigation is lesser in comparison to the clinical assessment. (Table 1)

The mean value obtained on the clinical assessment was -18.0 \pm 13.5 , while that obtained on the $\mathrm{x}$-ray is $-14.05 \pm 9.78$. The difference was found to be statistically not significant ( $\mathrm{P}>0.05)$ showing a comparable varus deformity shown on both clinical as well as on X-ray. (Table 1)

The mean value obtained on the navigation was $-9.28 \pm 8.66$, while that obtained on the $\mathrm{x}$-ray is $-14.05 \pm 9.78$. The difference was found to be statistically significant $(P<0.05)$ showing a lower mean deformity on the navigation in comparison to the $\mathrm{x}$-ray. (Table 1)

\section{After the surgery}

The mean value obtained on the clinical assessment was 4.90 \pm 7.38 , while that obtained on the navigation is $-0.74 \pm 2.66$. The difference was found to be statistically significant $(P<0.05)$ with a higher mean as measured clinically in comparison to the lower mean as seen on the navigation. The variance as seen on the navigation is lesser in comparison to the clinical assessment. Mean deformity on navigation is significantly low in comparison to clinical assessment. (Table 1)

The mean value obtained on the clinical assessment was 4.90 \pm 7.38 , while that obtained on the $x$-ray is $2.33 \pm 4.84$. The difference was found to be statistically significant $(P<0.05)$ with a higher mean as measured clinically in comparison to the lower mean as seen on the X-ray. The variance as seen on the X-ray is lesser in comparison to the clinical assessment. Mean deformity on X-ray is significantly low in comparison to clinical assessment. (Table 1)

The mean value obtained on the $\mathrm{x}$-ray assessment was $2.33 \pm$ 4.84 , while that obtained on the navigation was $-0.74 \pm 2.66$. The difference was found to be statistically significant $(P<0.05)$ with a higher mean as measured on $\mathrm{X}$-ray in comparison to the lower mean as measured by navigation. The variance as seen on the navigation is lesser in comparison to the $\mathrm{x}$-ray. Postoperative radiograph and navigation alignment discrepancy in the mechanical axis is seen. (Table 1)

\section{Before and after surgery comparisons}

Clinically, majority of the patients were having varus preoperatively, while postoperatively it was valgus. The mean pre-clinical value was $-18.03 \pm 13.46$, while post-clinical value was $4.90 \pm 7.38$. The difference was found to be statistically significant $(P<0.05)$, showing higher mean deformity preoperatively in comparison to postoperatively $(P<0.05)$. (Table 2)

On $\mathrm{x}$-ray, majority of the patients were having varus preoperatively, while postoperatively it was valgus. The mean pre-X-ray value was $-14.05 \pm 9.78$, while post-X-ray value was $2.33 \pm 4.84$. The difference was found to be statistically significant $(P<0.05)$, showing higher mean deformity preoperatively in comparison to postoperatively $(P<0.05)$. (Table 2)

On navigation, majority of the patients were having varus preoperatively as well as postoperatively. The mean prenavigation value was $-9.28 \pm 8.66$, while post-X-ray value was $-0.74 \pm 2.66$. The difference was found to be statistically significant $(P<0.05)$, showing higher mean deformity preoperatively in comparison to postoperatively $(P<0.05)$. (Table 2)

\section{Regression Lines}

The plotting of regression line and placements of points of values between the pairs pre-navigation and pre-clinical; pre$\mathrm{x}$-ray and pre-clinical; pre-navigation and pre-X-ray; postnavigation and post-clinical; post-X-ray and post-clinical; post-navigation and post-x-ray; pre-clinical and post-clinical; pre-X-ray and post-x-ray and pre-navigation and postnavigation are not in agreement with respect to assessment of deformity values in the patients undergoing primary total knee arthroplasty. Majority of points are far away from the regression line showing the non-agreement between the deformity values of given pairs. (Fig. 1,2)

\section{Conclusion}

The advantages of navigation in TKA include: improved restoration of the joint line, a reduction in peri-operative blood loss, better results in patients with extra-articular deformities and it has an important role in surgical training. Disadvantages include a longer operative time and increased cost. Complications associated with navigation technique are rare and mostly related to pin tracks.

Better alignment should lead to an improved functional outcome and an increased long-term survival of the prosthesis. As noted before, several studies have proven an improved function in the short and mid-term, while others found no significant differences. We still lack a long-term data regarding functional outcome and longevity of the prostheses.

\section{References}

1. Chang CW, Yang CY. Kinematic Navigation in Total Knee Replacement - Experience from the First 50 Cases. J Formos Med Assoc. 2006; 105(6):468-474.

2. Pang $\mathrm{CH}$, Chan WL, Yen $\mathrm{CH}$, Cheng SC, Woo SB, Choi ST et al. Comparison of total knee arthroplasty using computer-assisted navigation versus conventional guiding systems: a prospective study. J Orthop Surg (Hong Kong). 2009; 17(2):170-3.

3. Cooke TD, Scudamore RA, Bryant JT, Sorbie C, Siu D, Fisher B. A quantitative approach to radiography of the lower limb. Principles and applications. J Bone Joint Surg Br. 1991; 73:715-20.

4. Cooke TD, Sled EA, Scudamore RA. Frontal plane knee alignment: a call for standardized measurement. J Rheumatol. 2007; 34:1796-801.

5. Hauser R. Computer-aided 3D-navigation systems: a plea 
for an error model. HNO. 2000; 2:71-74.

6. Oberst M, Bertsch C, Lahm A, Wuerstlin S, Holz U. Regression and correlation analysis of preoperative versus intraoperative assessment of axes during navigated total knee arthroplasty. Comput Aided Surg. 2006; 11:8791.

7. Picard F, Gregori A, Leitner F. Computer Assisted Total Knee Arthroplasty: Validation of the Image Free Concept. Berlin, Germany: Pro Business, 2007. 